\title{
Studying the Stability of Some Non-Linear Time Series Models with Application
}

\author{
Abdulghafoor Salim \\ drabdul_salim@uomosul.edu.iq \\ College of Computer Science and \\ Mathematics, \\ University of Mosul,
}

\author{
Nihad Sharif Khalaf
}

College of Basic Education

University of Tikrit,

Accepted on: 04/03/2008

\section{ABSTRACT}

In this paper we study the stability of time series models in general, and for some non-linear time series models as a special case. Lagrange method to find the stability of non-linear models has been given.

The Leishmaniasis time series was studied and modeled by different non-linear models such as, seasonal ARIMA model by using the logarithmic transformation, exponential model of order two and the polynomial model. The stability of all such models by the above method has been obtained. From the comparison we find that the SARIMA is the best among all such models which we used for forecasting one year ago.

Keywords: stability, time series, non-linear time series models, Lagrange method.

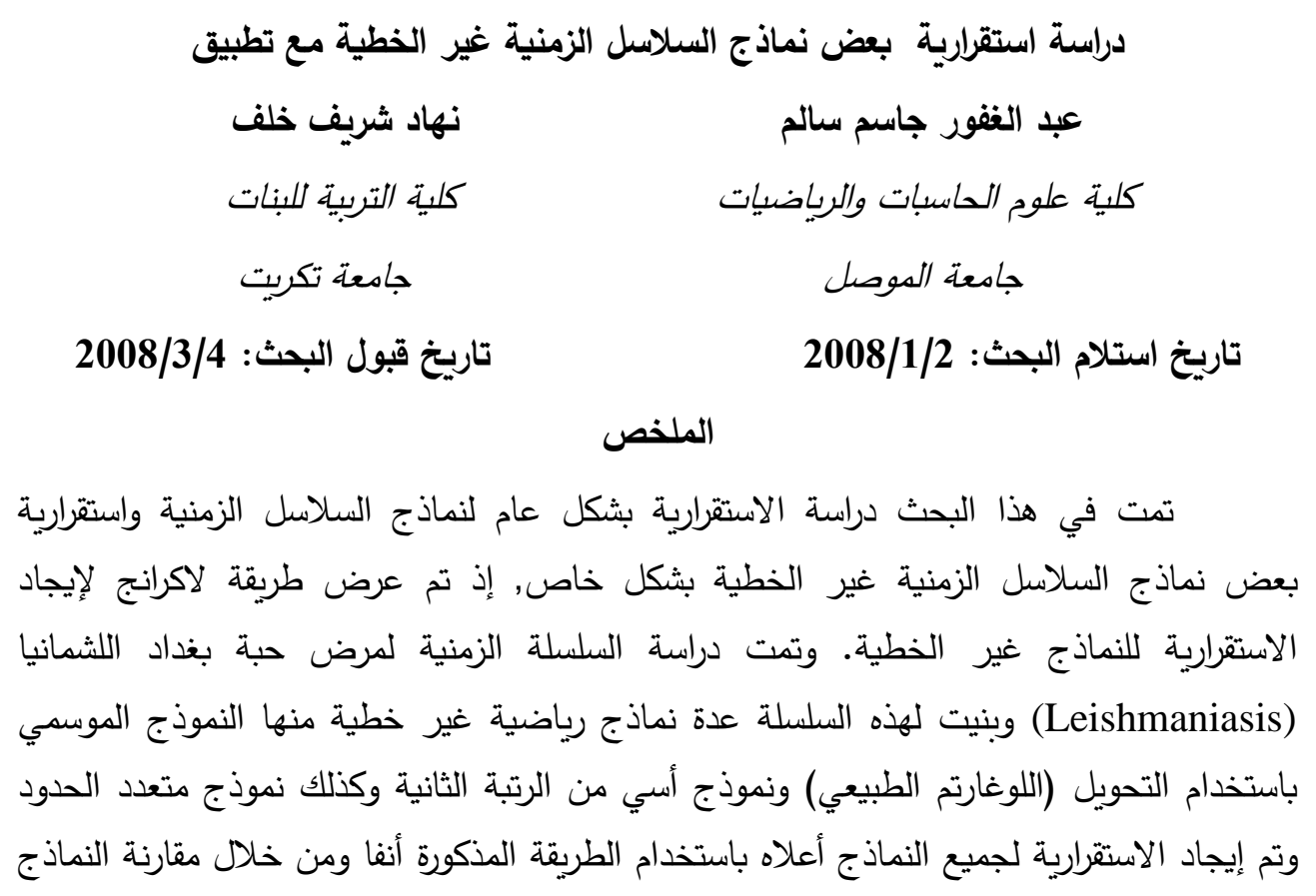

الملخص

تمت في هذا البحث دراسة الاستقرارية بشكل عام لنماذج السلاسل الزمنية واستقرارية

بعض نماذج السلاسل الزمنية غير الخطية بشكل خاص, إذ تم عرض طريقة لاكرانج لإيجاد

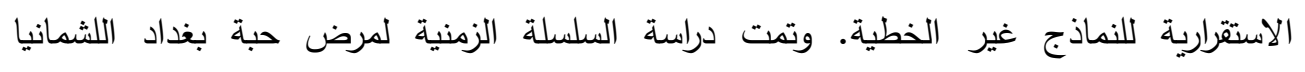

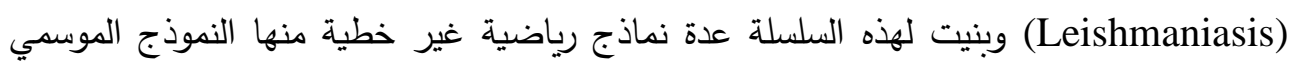

باستخدام التحويل (اللوغارتم الطبيعي) ونموذج أسي من الرتبة الثانية وكذلك نموذج متعدد الحدود

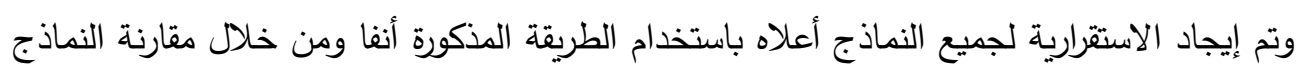


أعلاه تبين أن النموذج الموسمي المندمج يكون أفضل النماذج المقترحة لتمثيل السلسلة وتم التتبؤ بالقيم المستقبلية للمرض ولفترة سنة واحدة باستخدام النموذج الموسيمي. الكلمات المفتاحية: الاستقرارية، السلاسل الزمنية، نماذج غير الخطية، طريقة لاكرانج.

Introduction : المقدمة

تبنى السلاسل الزمنية عادة على الافتراضات آلاتية: الاستقرارية ، الخطية ، الطبيعية علماً إن اغلب

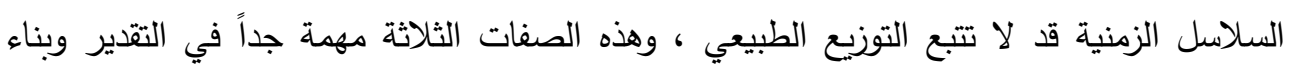

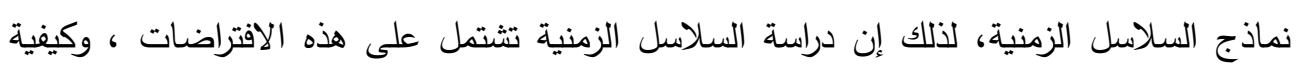

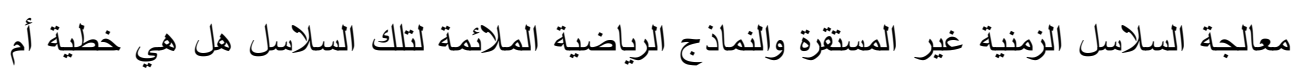

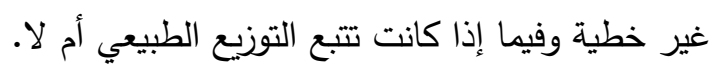

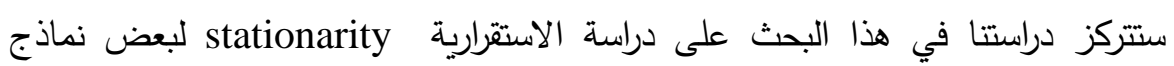
السلاسل الزمنية غير الخطية .

تتاول Priestley, M.B. في عام (1988) دراسة عدم استقرارية النماذج غير الخطية للسلاسل الزمنية [18] وفي عام (1990) قام . Tong, H. بدراسة النظام الديناميكي مع الاستقرارية Bongeral, and Picard, N. في السلاسل الزمنية غير الخطية [21] وفي عام (1992) تتاول

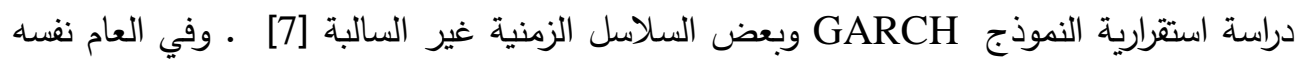
بحث Brockwell, P.J. and Tweedie, R.L. وجود الاستقرارية في نماذج العتبة للنموذج

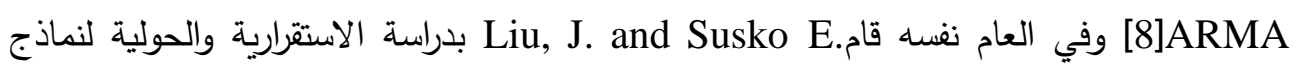
ARMA عير الخطية [12]وفي العام نفسه درس Manncar عدم الاستقرارية في نماذج السلاسل الزمنية [11] وفي 1993 درس Meyn, S.P. and Tweedie, R.L. ملاسل ماركوف والاستقرارية التصادفية [13] وفي عام (1996) درس Manuca, R. and Savit, R سلاركية

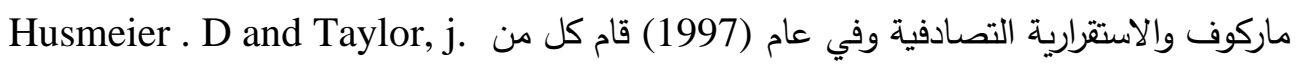

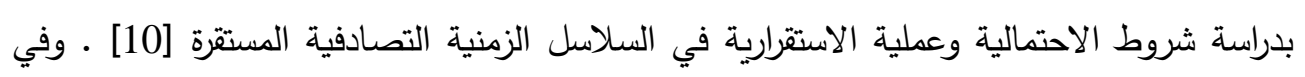
العام نفسه قام Schreiber بتحليل عدم الاستقرارية في السلاسل الزمنية [18] وفي العام نفسه قام لعام

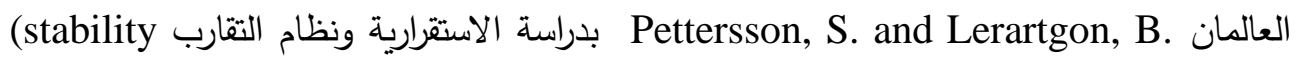

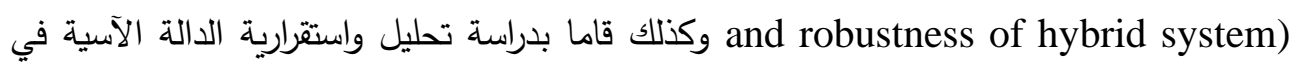

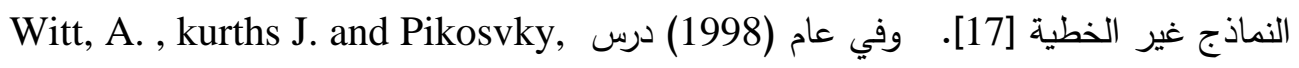

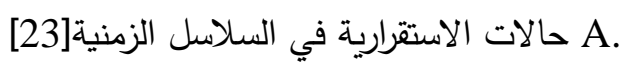




\section{Stationary and Stability المرحلية و الاستقرارية}

تصادفنا في كثير من المسائل الفيزيائية والهندية عمليات يمكن وصفها بأنها في حالة اتزان إحصائي Statistical Equilibrium ومعنى ذلك إننا لو حصلنا على مشاهدات لعملية من هذا النوع وتم تقسيمها إلى مجاميع من الفترات الزمنية فان المقاطع المختلفة لهذه المشاهدات

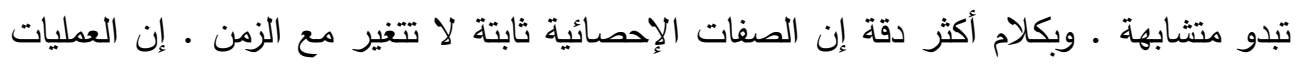

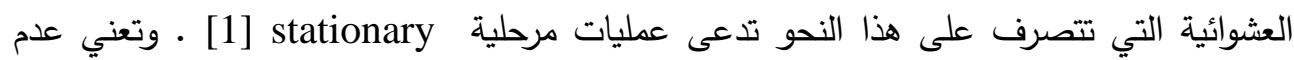

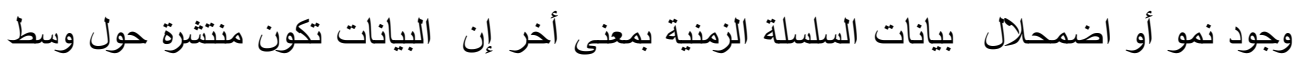
ثابت ولها تباين ثابت .فان ذلك يعني نفسها أي إن f(x) حيث f الاحتمالي المشترك لا يتغير مع تغير الفترة الزمنية أو عند الإزاحة بأعداد ثابتة . نتيجة [1]] : إن العمليات المرحلية Stationary تتشأ عادة من خلال نظام مستقر stable يصل إلى حالة الثبات Steady state بعد فترة زمنية مناسبة. وقد عرف Wei (1990) النظام المستقر (stable)

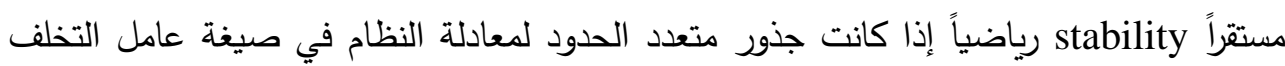
(lag operator) كلها داخل دائرة الوحدة [1] .

Stationarity of non Linear Models [21][15][6] 2-1 استقرارية النماذج غير الخطية إن التقنيات المطورة لدراسة الاستقرارية وهي استقرارية نماذج السلاسل الزمنية الخطية، تعتمد اعتمادا كبيرا على الافتراضية الخطية ولذلك لا يمكن إن نتوسع بسهولة إلى الحالة غير

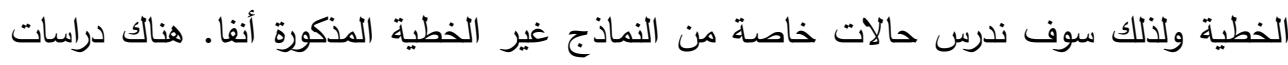
عديدة لإيجاد الاستقرارية للنماذج غير الخطية منها إيجاد الاستقرارية وحسب طريقة لبئ لبينوف المباشرة

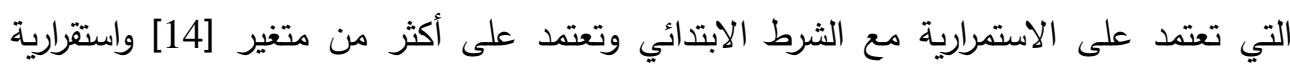
بويسون ( لاحظ المصدر نفسه ). وكذلك استقرارية لاكرانج التي عادة تقتصر على السلسلة أو عدة سلاسل ، ويجب إن تكون السلسلة محددة أي

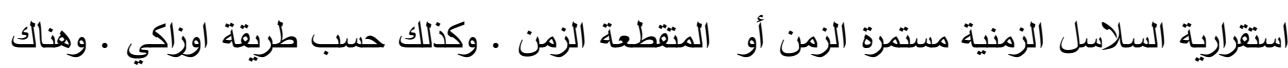
دراسات عديدة في هذا المجال ( Tong 90 ) وسوف يتركز اهتمامنا على استقرارية لاكرانج استقرارية اوزاكي ولزيادة في التفاصيل لاحظ [2]. 
2-2 الاستقرارية حسب طريقة لاكرانج

هناك عدة دراسات لإيجاد استقرارية بعض النماذج غير الخطية منها النماذج الآسية (لاحظ [15]) الاستقرارية للنماذج الثنائية (Bilinear Model) (لاحظ [22]) وإيجاد الاستقرارية لنماذج العتبة للانحدار الذاتي ( لاحظ [21]) • معظم هذه الدراسات تستخدم التقريب الخطي لهذه النماذج غير الخطية ـ أي انه يمكن تحويل النماذج غير الخطية إلى صيغة مشابهة لصيغة النماذج الخطية وبافتراضات معينة فعلى سبيل

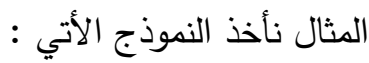

$X_{t}=a_{1} X_{t-1}+a_{2} X_{t-2}+\pi X_{t-1}^{3}+Z_{t}$

هذا النموذج يمثل نموذج متعدد حدود وهو نموذج غير خطي يكتب هذا النموذج بالثكل الأتي :

$$
X_{t}=\left(a_{1}+\pi X_{t-1}^{2}\right) X_{t-1}+a_{2} X_{t-2}+Z_{t}
$$

وهو نموذج انحدار ذاتي من الرتبة الثانية وبوضع قيود معينة على معامل $X_{t-1}$ يمكن إيجاد الاستقرارية بصورة مشابهة للنماذج الخطية . لمودية سوف نذكر بعض التعارف والمبرهنات الأساسية التي تحتاج اليها في إيجاد الاستقرارية بطريقة • لاكرانج

\section{[21] مبرهنة (1-1)} لتكن $\xi=\left\{\begin{array}{lll}A \xi_{t-1} & \text { if } & X_{t-2}>0 \\ B \xi_{t-1} & \text { if } & X_{t-2} \leq 0\end{array}\right.$

$$
B=\left[\begin{array}{ll}
\phi^{\prime} & 0 \\
1 & 0
\end{array}\right] \quad, \quad A=\left[\begin{array}{ll}
\phi_{1} & \phi_{2} \\
1 & 0
\end{array}\right] \quad, \quad \xi=\left[\begin{array}{l}
X_{t} \\
X_{t-1}
\end{array}\right]
$$

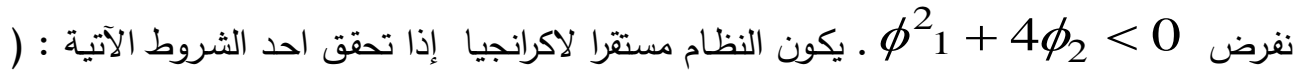
لاحظ [21] 


$$
\begin{aligned}
& 1-\quad \phi_{1}^{\prime}<0 \quad, \phi_{1} \geq 0 \quad \text { and } \quad \phi_{1}^{\prime}\left(\phi_{1} \phi_{1}^{\prime}+\phi_{2}\right) \leq 1 \\
& 2-\quad \phi_{1}^{\prime}<0 \quad, \phi_{1}<0 \quad, \phi_{1} \phi_{1}^{\prime}+\phi_{2}<0 \quad \text { and } \quad \phi_{1}^{\prime}\left(\phi_{1} \phi_{1}^{\prime}+\phi_{2}\right) \leq 1 \\
& 3-\quad \phi_{1}^{\prime}<0 \quad, \phi_{1}<0 \quad \text {, and } \quad \phi_{1} \phi_{1}^{\prime}+\phi_{2}=0 \\
& 4-\quad \phi_{1}^{\prime}<0 \quad, \phi_{1}<0 \quad \phi_{1} \phi_{1}^{\prime}+\phi_{1} \quad \text {, and } \quad \phi_{1} \phi_{1}^{\prime}+\phi_{2} \leq 1 \\
& 5-\quad \phi_{1}^{\prime}=0 \\
& 6-\quad 1 \geq \phi_{1}^{\prime}>0 \quad, \phi_{1} \geq 0 \\
& 7-\quad 1 \geq \phi_{1}^{\prime}>0 \quad, \phi_{1}<0 \quad,\left(\phi_{1}^{2}+\phi_{2}\right) \phi_{1}^{\prime}+\phi_{1} \phi_{2} \leq 0 \\
& 8-\quad 1 \geq \phi_{1}^{\prime}>0 \quad, \phi_{1}<0 \quad,\left(\phi_{1}^{2}+\phi_{2}\right) \phi_{1}^{\prime}+\phi_{1} \phi_{2}>0 \\
& \text { and } \quad\left(\phi_{1}^{2}+\phi_{2}\right) \phi_{1}^{\prime}+\phi_{1} \phi_{2} \leq 1
\end{aligned}
$$

[15][14] (A singular point) (النقطة المنفردة ) 1-1

لتكن

$$
X_{t}=F\left(X_{t-1}, \ldots, X_{t-p}\right)
$$

تعرف النقطة المنفردة $\xi$ للمعادلة (4-1) بأنها تلك النقطة التي يقترب منها مسار المعادلة عندما

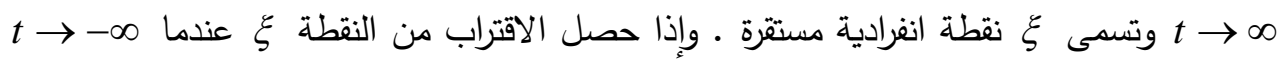
فان النقطة $\xi$ تسمى نقطة انفرادية غير مستقرة .

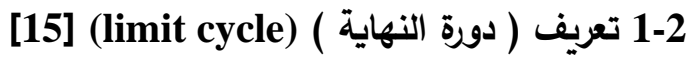

$$
X_{t}=F\left(X_{t-1}, \ldots, X_{t-p}\right)
$$

$$
X_{t}, X_{t+1}, X_{t+2}, \ldots, X_{t+q}=X_{t}
$$

$$
\text { حيث q عدد صحيح موجب يمثل الدورة . }
$$

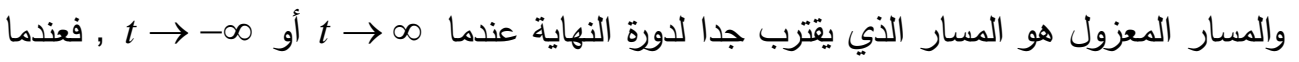

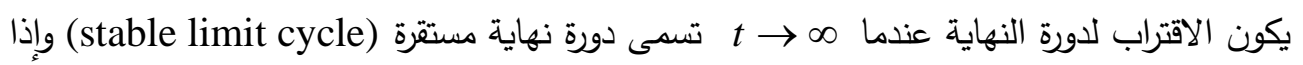

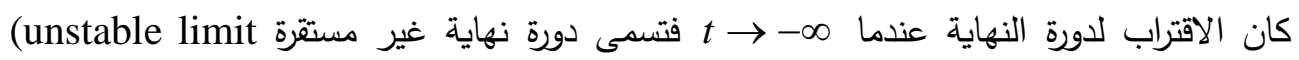
cycle) 
$\left(X_{1+k q}, \ldots, X_{p+k q}\right)=\left(X_{1}, \ldots, X_{p}\right)$

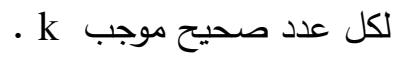

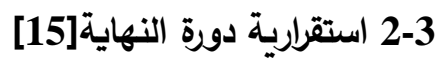

سوف نبدأ بتوضيح استقرارية دورة النهاية على نموذج غير خطي أسي من الرتبة الأولى ثم نعمم ألفكره . أنمون.

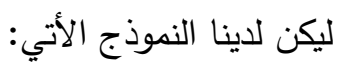
$X_{t}=\left(\phi_{1}+\pi_{1} e^{-X_{t-1}^{2}}\right) X_{t-1}+\varepsilon_{t}$

حيث تكون لها دورة نهاية معلومة yt ب بالقرب من النقطة $x_{t}$ الواقعة على المسار والقرببة من دورة

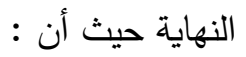
$y_{t}, y_{t+1}, \ldots . y_{t+q-1}, y_{t+q}=y_{t}$

$X_{s}=y_{s}+\xi_{s}$ حيث |ظ| صغيرة ضمن الفترة النهاية يمكن إيجادها كما يأتي: $x_{t-1}=y_{t-1}+\xi_{t-1} \quad$ and $\quad X_{t}=y_{t}+\xi_{t}$

نعوض في المعادلة (5-1) وبإجراء بعض العمليات الجبرية البسيطة نحصل على ما ياتي: $\xi_{t}=\left\{\phi_{1}+\pi_{1}\left(1-2 y_{t-1}^{2}\right) e^{-y_{t-1}^{2}}\right\} \xi_{t-1}+O\left(\xi_{t-1}\right)$

ومنها نستطيع ملاحظة انه إذا كان الحل للمعادلة الآتية: $\xi_{t}=\left\{\phi_{1}+\pi_{1}\left(1-2 y_{t-1}^{2}\right) e^{-y_{t-1}^{2}}\right\} \xi_{t-1}$

يقترب من الصفر عندما ما $t$ تكون دورة النهاية مستقرة. وبما أن المعادلة (7-1) هي معادلة فرقية خطية وبمعاملات دورية يصعب حلها تحليليا لذا يمكن

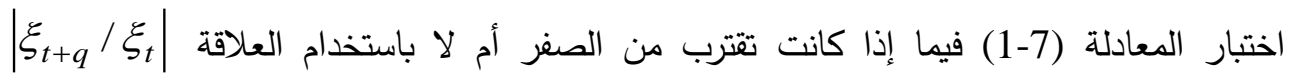
وتقترب $\xi_{t}$ من الصفر إذا كانت هذه العلاقة اقل من الواحد. 


$$
\begin{aligned}
& \text { من المعادلة (7-1) يمكن الحصول على } \\
& \xi_{t+q}=\left\{\phi_{1}+\pi_{1}\left(1-2 y_{t+q-1}^{2}\right) e^{-y_{t+q-1}^{2}}\right\} \xi_{t+q-1} \\
& =\left\{\phi_{1}+\pi_{1}\left(1-2 y_{t+q-1}^{2}\right) e^{-y_{t+q-1}^{2}}\right\}\left\{\phi_{1}+\pi_{1}\left(1-2 y_{t+q-2}^{2}\right) e^{-y_{t+q-2}^{2}}\right\} \xi_{t+q-2} \\
& \text { نستمر بهذه الطريقة لنحصل على ما يأتي: } \\
& \xi_{t+q}=\prod_{i=1}^{q}\left\{\phi_{1}+\pi_{1}\left(1-2 y_{t+i-1}^{2}\right) e^{-y_{t+i-1}^{2}}\right\} \xi_{t}
\end{aligned}
$$

وعليه يكون |

$\left|\frac{\xi_{t+q}}{\xi_{t}}\right|=\mid \prod_{i=1}^{q}\left\{\phi_{1}+\pi_{1}\left(1-2 y_{t+i-1}^{2}\right) e^{-y_{t+i-1}^{2}}\right\}$

ولاستخدام المعادلة (1-1) ) يمكن الاستفادة من مبرهنة الاستقرارية آلاتية.

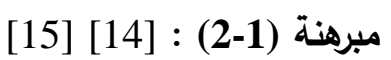

دورة النهاية بدورة q للنموذج (1-1-1) تكون مستقرة إذا كان:

$\left|\xi_{t+q} / \xi_{t}\right|<1$

البرهان: (لاحظ [14] )

باستخدام الطريقة أعلاه والمبرهنة (1-3) الآتية يمكن أيجاد استقرارية دورة النهاية للنموذج الآسي

$$
X_{t}=\left(\phi_{1}+\pi_{1} e^{-X_{t-1}^{2}}\right) X_{t-1}+\varepsilon_{t}
$$

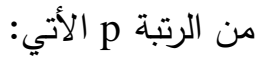

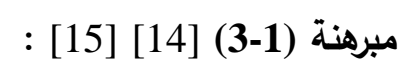

دورة النهاية بدورة للنموذج الآسي (9-1) تكون مستقرة إذا كانت القيمة المطلقة للقيم

الذاتية للمصفوفة اقل من الواحد بالقيمة المطلقة، بحيث:

$$
\begin{aligned}
A & =A_{q} \cdot A_{q-1} \cdot \ldots \cdot A_{1} \\
A_{i} & =\left[\begin{array}{ccccc}
a_{1.1}^{(i)} & a_{1.2}^{(i)} & \cdots & a_{1 . p^{-1}}^{(i)} & a_{1 . p}^{(i)} \\
1 & 0 & \cdots & 0 & 0 \\
0 & 1 & \cdots & \vdots & \vdots \\
\vdots & \ddots & \cdots & & \\
0 & \cdots & 0 & 1 & 0
\end{array}\right]
\end{aligned}
$$


وأن

$a_{1.1}^{(i)}=\phi_{1}+\pi_{1}-2 \sum_{j=1}^{p}\left(\pi_{j} \bar{y}_{t+i-j}\right) \bar{y}_{t+j-i} e^{-\bar{y}_{t+i-1}^{2}}$

$a_{i, k}^{(i)}=\phi_{k}+\pi_{k} e^{-\bar{y}_{t+i-1}^{2}}, \quad k=2,3, \ldots p$

ملاحظة [14] : إن استقرارية النقاط المنفردة غير الصغرية للنموذج (9-1) يمكن اختبارها بسهولة

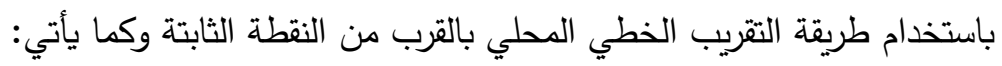
لتكن X مغلقة للنقطة المنفردة غير الصفرية گ والتي يمكن إيجادها من العلاقة $X_{t}=\xi+\xi_{t}$

حيث

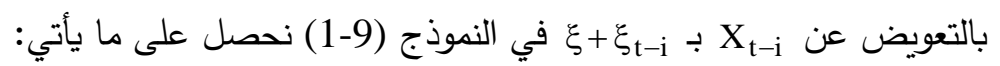
$\xi_{\mathrm{t}}=\mathrm{h}_{1} \xi_{\mathrm{t}-1}+\mathrm{h}_{2} \xi_{\mathrm{t}-2}+\ldots+\mathrm{h}_{\mathrm{p}} \xi_{\mathrm{t}-\mathrm{p}}$

$h_{1}=\frac{\Pi_{1}+\phi_{1} \sum_{j=1}^{p} \Pi_{j}-\Pi_{1} \sum_{j=1}^{p} \phi_{j}}{\sum_{j=1}^{p} \Pi_{j}}+2\left(1-\sum_{j=1}^{p} \phi_{j}\right) L n\left(\frac{1-\sum_{j=1}^{p} \phi_{j}}{\sum_{j=1}^{p} \Pi_{j}}\right)$

$h_{i}=\frac{\Pi_{i}+\phi_{i} \sum_{j=1}^{p} \Pi_{j}-\Pi_{i} \sum_{j=1}^{p} \phi_{j}}{\sum_{j=1}^{p} \Pi_{j}} \quad ; \quad i=2,3 \ldots p$

إن الثرط الضروري والكافي لـ گ لتتترب من الصفر هو أن تكون جذور المعادلة الميزة للمعادلة (12) - تقع داخل دائرة الوحدة.

الجانب التطبيقي PRACTICAL PART

تناولنا في هذا البحث بيانات احد الأمراض الثائعة في الثرق الأوسط عموما وفي العراق

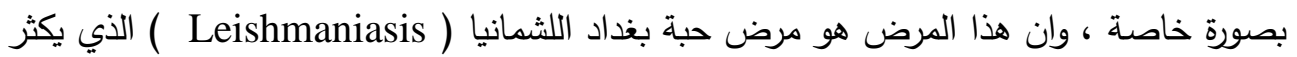

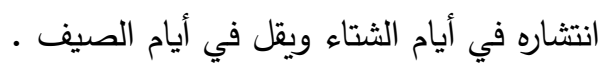

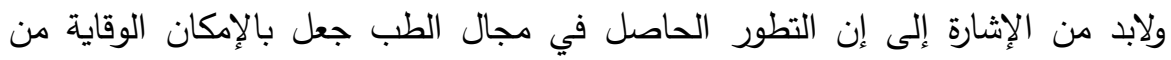

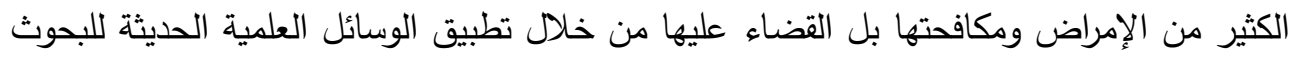

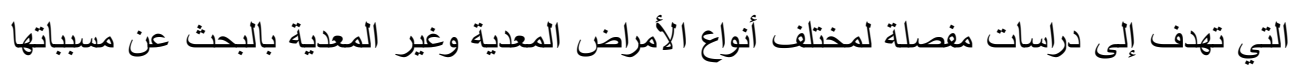


وطرائقها تكاثرها بين أفراد المجتمع لغرض التعرف على طرائق الوقاية منها والسيطرة عليها ومنع انتشارها [ [9].

وتمت نمذجة السلسلة بنماذج خطية منها ARMA,AR,MA,SARIMA

وباستخدام البرنامج الجاهز وبالاعتماد على اقل مربع خطأ MSE حصلنا على النموذج الموسمي

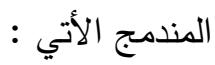

SARIMA $(2,0,0)(3,0,0) 12 \mathrm{y}_{\mathrm{t}}$

حيث

$\Phi_{1}=0.73392$

std.Err 0.07257

$\Phi_{2}=0.14482$

0.06688

وان معاملات الانحدار الذاتي للجزء الموسمي هي:

$\Phi_{1}=0.31155$

std.Err 0.7175

$\Phi_{2}=0.18983$

0.07087

$\Phi_{3}=0.22217$

0.06960

Plot of variable: VAR 1

$\ln (x)$; ARIMA $(2,0,0)(3,0,0)$ residuals;

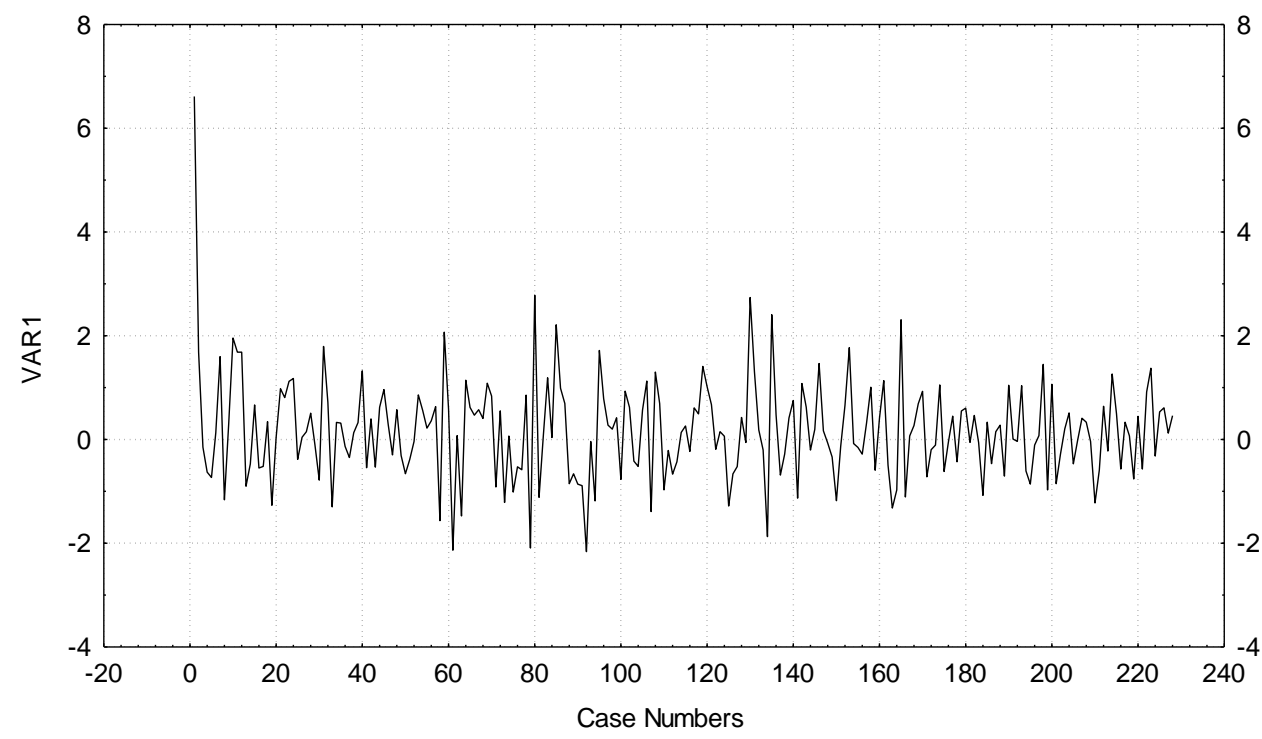

الشكل (1-1) يمثل رسم البواقي للنموذج الموسمي (3,0,0) (2,0,0) باستخدام اللوغاريتم 
Autocorrelation Function

VAR1 : In $(x)$; ARIMA $(2,0,0)(3,0,0)$ residuals:

(Standard errors are white-noise estimates)
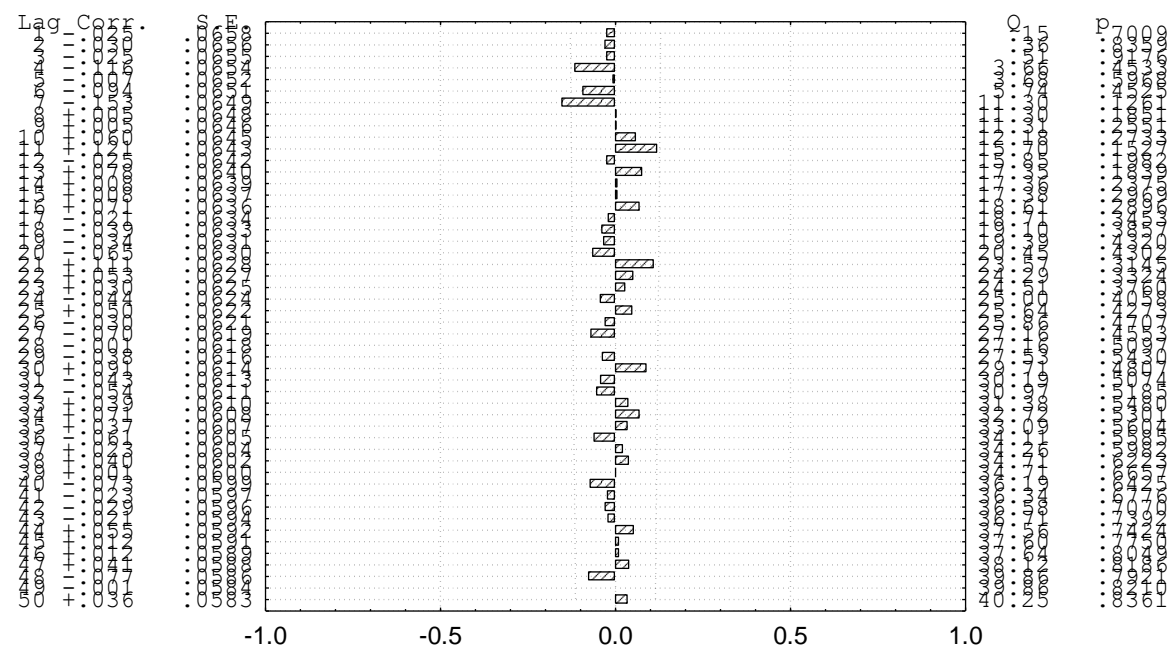

الثكل (2-1) يمثل رسم دالة الارتباط الذاتي للنموذج الموسمي (3,0,0) (2,0,0) باستخدام

Normal Probability Plot: VAR1

$\ln (\mathrm{x})$; ARIMA $(2,0,0)(3,0,0)$ residuals;

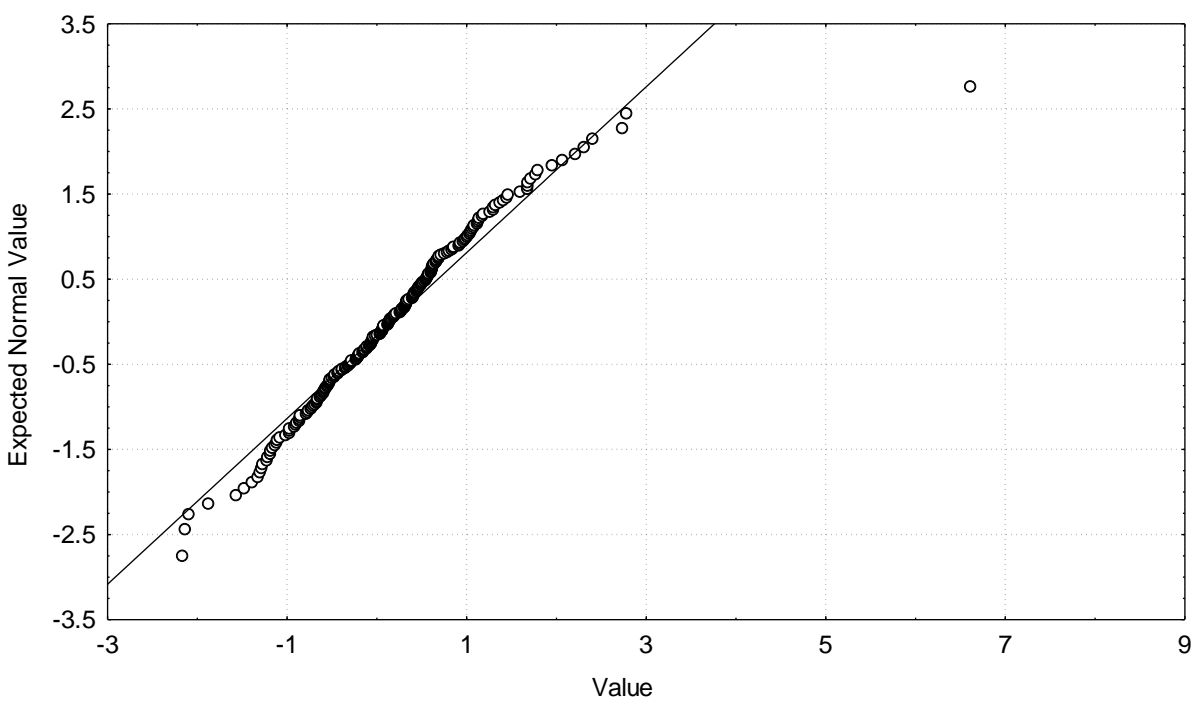

الثكل (3-1) يمثل رسم الاحتمالية الطبيعية لنموذج الموسمي (3,0,0) (2,0,0) باستخدام

اللوغاريتم 
الثكل (1-1) يمثل رسم البواقي للنموذج الموسمي المضاعف باستخدام اللوغاريتم ، الثكل (2-1) يمثل رسم دالة الارتباط الذاتي لبواقي النموذج الموسي المضاعف (3,0,0) (2,0,0) باستخدام

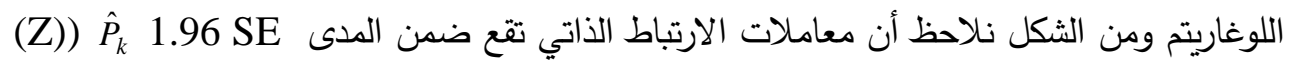
干 ) وباحتمالية 95\% وأنها غير مترابطة Uncorrelated. ومن الثكل (3-1) نلاحظ أن البواقي تتبع التوزيع الطبيعي مما يشير إلى ملائمة النموذج وهو النموذج الأفضل من بقية وانية النماذج.

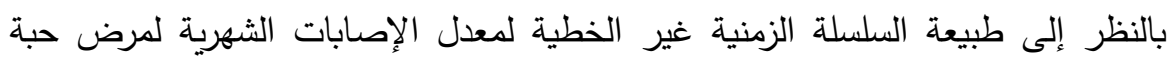

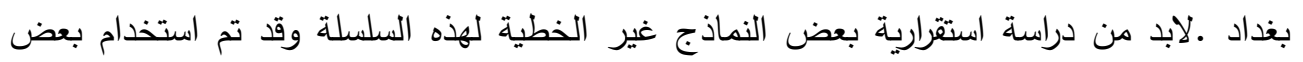

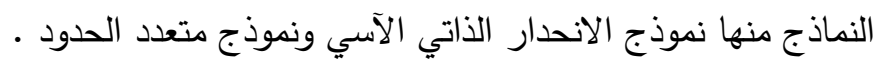

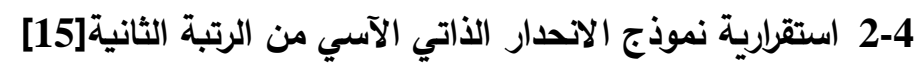
والذي يعرف بالصيغة آلاتية : $X_{t}=\left(\phi_{1}+\pi_{1} e^{-\gamma x^{2} t-1}\right) X_{t-1}+\left(\phi_{2}+\pi_{2} e^{-r x_{t-1}^{2}}\right) X_{t-2}+Z_{t}$ $X_{t}=\left(\phi_{1}+0.01 e^{-10 x^{2} t-1}\right) X_{t-1}+\left(\phi_{2}+0.02 e^{-20 x_{t-1}^{2}}\right) X_{t-2}+Z_{t}$

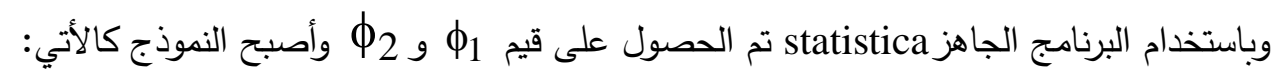
$X_{t}=\left(0.789+0.01 e^{-10 x^{2} t-1}\right) X_{t-1}+\left(0.0218+0.02 e^{-20 x_{t-1}^{2}}\right) X_{t-2}+Z_{t}$ ولدراسة استقرارية النموذج لابد من إيجاد قيم $\lambda_{2} \lambda_{2}$ $e^{x_{t-1}^{2}} \longrightarrow 0$ فأن $x_{t-1}^{2} \longrightarrow 1$ أولا: عندما فتصبح المعادلة كالآتي:

$\lambda^{2}-0.729 \lambda-0.04=0$ $\lambda_{1}=-0.0513 \quad \lambda_{2}=0.7803$

$e^{x_{t-1}^{2}} \longrightarrow 0 \quad x_{t-1}^{2} \longrightarrow \infty \quad$ عندما $X_{t}=\left(0.789+0.01 e^{-10 x^{2} t-1}\right) X_{t-1}+\left(0.0218+0.02 e^{-20 x_{t-1}^{2}}\right) X_{t-2}+Z_{t}$ باستخدام المعادلة المميزة تصبح المعادلة كما يأتي:

$\lambda^{2}-0.789 \lambda-0.0218=0$

$\lambda_{1}=-0.0267$

$\lambda_{2}=0.8157$

لتوضيح فيما إذا كانت النقطة المنفردة تحقق شرط الاستقرارية نستخدم الملاحظة المذكورة أنفا إذ يجب أن تتحقق العلاقة الآتية: ليما 
$\xi_{t}=h_{1} \xi_{t-1}+h_{2} \xi_{t-2}$

$\lambda^{2}-h_{1} \lambda-h_{2}=0$

والتي معادلتها المميزة هي:

لتوضيح فيما إذا كانت النقطة المنفردة تحقق شرط الاستقرارية نستخدم الملاحظة المذكورة أنفا إذ يجب أن تتحقق العلاقة الآتية: لئحا

$\xi_{\mathrm{t}}=\mathrm{h}_{1} \xi_{\mathrm{t}-1}+\mathrm{h}_{2} \xi_{\mathrm{t}-2}$

والتي معادلتها المميزة هي:

$\lambda^{2}-\mathrm{h}_{1} \lambda-\mathrm{h}_{2}=0$

وبالتعويض عن قيم المعاملات $\pi_{i}, \phi_{i}$

$\mathrm{h}_{1}=0.3611$

على ما يأتي:

$\mathrm{h}_{2}=0.019$

نجد جذور المعادلة الآتية:

$\lambda^{2}-h_{1} \lambda-h_{2}=0$

$\lambda_{1}=-0.0466$

$\lambda_{2}=0.4077$

ومن هذا نستنتج أن الجذور اقل من واحد

أذن يكون النموذج الآسي من المرتبة الثانية مستقراً.

2-5 استقرارية النموذج متعدد الدودد:

\section{POLYNOMIAL MODEL STATIONARTY}

$$
\begin{aligned}
& \text { يعرف نموذج الانحدار الذاتي المتعدد الحدود بالصيغة آلاتية : } \\
& x_{t}=\phi_{1} x_{t-1}+\ldots .+\phi_{p} x_{t-p}+Q\left(x_{t-1}, \ldots, x_{t-p}\right)+z_{t} \\
& \text { حيث أن } \\
& \text {. . } x_{t-1} \ldots x_{t-p} \\
& \text { باستخدام البرنامج الجاهز statistica حصلنا على النموذج الأتي: } \\
& X_{t}=0.879 X_{t-1}-0.000001 X_{t-1}^{3}+0.009588 X_{t-2}+Z_{t} \\
& X_{t}=\left(0.879-0.000001 X_{t-1}^{2}\right) X_{t-1}+0.009588 X_{t-2}+Z_{t}
\end{aligned}
$$

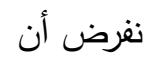

$$
\begin{aligned}
& \left(0.879-0.000001 X_{t-1}^{2}\right)=\left(0.879-0.000001-0.000001 e^{-x_{t-1}^{2}}\right)
\end{aligned}
$$




$$
\begin{aligned}
& \text { فيصبح النموذج } \\
& X_{t}=\left(0.879-0.000001-0.000001 e^{-x_{t-1}^{2}}\right) X_{t-1}+0.009588 X_{t-2}+Z_{t} \\
& \text { وهو نموذج آسي }
\end{aligned}
$$

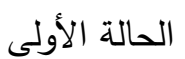

$$
\begin{aligned}
& \text { عندما } \\
& \longrightarrow e^{-x_{t-1}^{2}} 1 \longrightarrow x_{t-1}^{2} \quad \text { فأن } \quad 0 \\
& \mathrm{X}_{\mathrm{t}}=(0.8789) \mathrm{X}_{\mathrm{t}-1}+0.009588 \mathrm{X}_{\mathrm{t}-2}+\mathrm{Z}_{\mathrm{t}} \\
& \lambda^{2}-0.87899 \lambda-0.09588=0 \\
& \lambda_{1}=-0.0981 \\
& \lambda_{2}=0.9771
\end{aligned}
$$

الحالة الثانية

$$
e^{-x_{t-1}^{2}} \rightarrow 0 \text { فندما }
$$

$X_{t}=(0.87899) X_{t-1}-0.000001 X_{t-2}+Z_{t}$

وتكون قيم

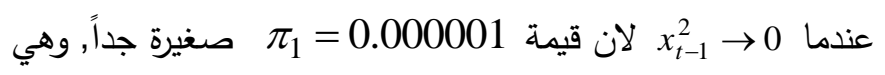
$\lambda_{1}=0.878990$

$\lambda_{2}=0.87899$

ومن الحالة الأولى والحالة الثانية نستنتج أن قيم متعدد الحدود مستقراً. والجدول آلاتي يمثل النماذج التي حصلنا عليها وصفاتها الإحصائية (معدل مربع الخطأ والانحراف القياسي و NBIC ) لكل منها.

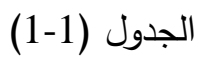

جدول مقارنة بين النماذج المقترحة

\begin{tabular}{|c|c|c|c|c|c|c|}
\hline $\begin{array}{c}\text { MODEL } \\
\text { STAT }\end{array}$ & AR(6) & MA (7) & ARMA(7,7) & SARIMA & EXP(AR(P)) & $\begin{array}{c}\text { POLEN- } \\
\text { OMIAL } \\
\text { MODEL }\end{array}$ \\
\hline \hline M.S.E & 14019 & 14469 & 11732 & 1.0124 & 28261 & 28383 \\
\hline \hline NBIC & 4.9272 & 4.9645 & 4.9815 & 0.1400 & 1.3540 & 3.8600 \\
\hline \hline S.D & 116.817 & 118.395 & 104.673 & 0.97923 & 6.4000 & 6.7010 \\
\hline
\end{tabular}


) ومن الجدول ومن ملاحظة دالة الارتباط الذاتي ورسم الاحتمالية الطبيعية لبواقي النماذج أعلاه

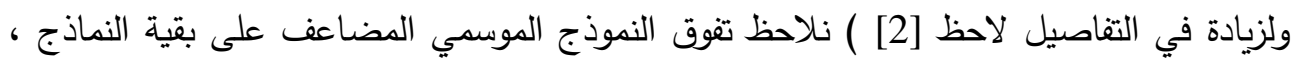
وبأقل معدل مربع خطأ وبأقل NBIC لذلك يكون هو النموذج الأفضل الذي يمثل السلسلة

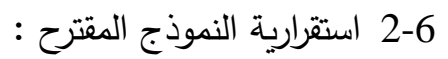
النموذج المقترح معادلته هو: SARIMA $(2,0,0)(3,0,0)_{12} y_{t}$
$\Phi_{1}$
$\Phi_{2}$
$\Phi_{s 1}$
$\Phi_{s 2}$
$\Phi_{s 3}$

$0.73392+0.14482+0.31155+0.18983+0.22217$

$\begin{array}{llllll}\text { StaErr } & 0.07257 & 0.06688 & 0.7175 & 0.07087 & 0.06960\end{array}$

يكون النموذج مستقرا إذا كانت جذور المعادلة المميزة للجزء غير الموسمي والجزء الموسمي تقع داخل دائرة الوحدة

$$
\begin{aligned}
& \lambda^{2}-0.7337 \lambda-0.144=0 \\
& \lambda_{1}=-0.1610 \\
& \lambda_{2}=0.8947
\end{aligned}
$$

$$
\begin{aligned}
& \lambda^{3}-0.3 \lambda^{2}-0.19 \lambda-0.2=0 \\
& \lambda_{1}=0.846 \\
& \lambda_{2}=-0.267+\mathrm{i} 0.4399 \\
& \lambda_{3}=-0.267-\mathrm{i} 0.4399 \\
& \theta_{2}=\tan ^{-1} \frac{y}{x}=-58.692 \\
& r_{2}=0.513 \\
& \theta_{3}=\tan ^{-1} \frac{y}{x}=58.692 \\
& r_{3}=0.5137
\end{aligned}
$$$$
\text { والمعادلة المميزة للجزء الموسمي هي }
$$$$
\text { المعادلة المميزة للجزء غير الموسي هي: }
$$

حيث نلاحظ أن المطلقة وكذلك الحال بالنسبة إلى جذور المعادلة المميزة للجزء غير الموسمي مما يشير إلى أن

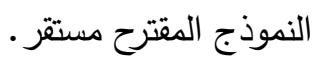


والجدول الأتي يمثل القيم المتتبأ بها من النماذج المذكورة آنفا:

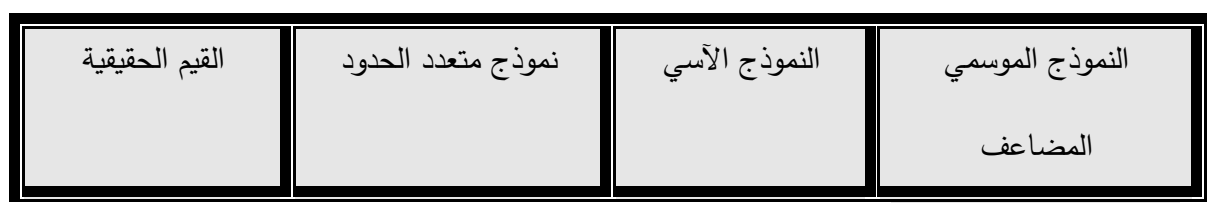

\begin{tabular}{|c|c|c|c|}
\hline 75 & 519 & 492 & 90 \\
\hline 80 & 214 & 124 & 86 \\
\hline 93 & 67 & 28 & 109 \\
\hline 80 & 22 & 10 & 75 \\
\hline 21 & 19 & 18 & 30 \\
\hline 10 & 63 & 73 & 21 \\
\hline 14 & 11 & 0 & 13 \\
\hline 15 & 13 & 13 & 14 \\
\hline 10 & 60 & 71 & 6 \\
\hline 33 & 152 & 172 & 9 \\
\hline 98 & 376 & 425 & 10 \\
\hline 64 & 499 & 516 & 19 \\
\hline
\end{tabular}

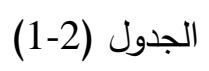

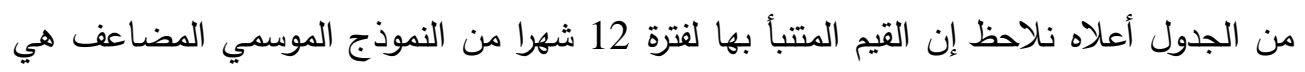

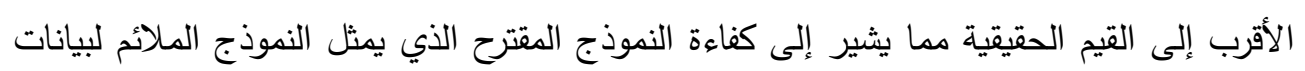

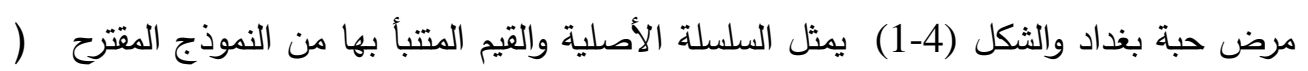
الموسمي المضاعف). 
Forecasts; Model:(2,0,0)(3,0,0) Seasonal lag: 12

Input: VAR1 $: \ln (\mathrm{x})$

Start of origin: 1 End of origin: 228

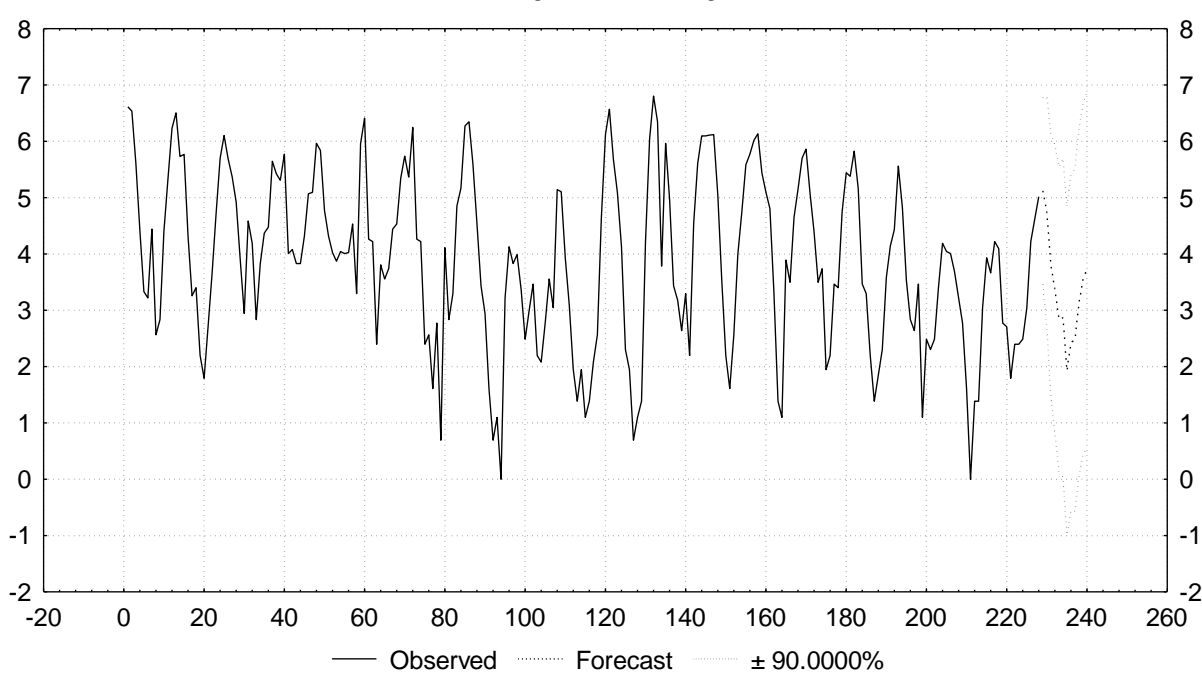

الشكل (4-1) يمثل رسم التكهن للسلسلة للنموذج (3,0,0) (2,0,0) باستخدام اللوغاريتم

الاستنتاجات

1- إن السلسلة الزمنية للإصابة بمرض حبة بغداد في العراق تبين أنها سلسلة غير مستقرة ولا يوجد اتجاه واضح للسلسلة .

2- إن النـوذج الموسـي المضــاعف من الرتبـة (3,0,0) (2,0,0) هو النــوذج الملائم للسلسـلة الزمنية للإصابة بمرض حبة بغداد وقد أعطى تقديرات جيدة ( للتبوء بالقيم المستقبلية) وقريبة

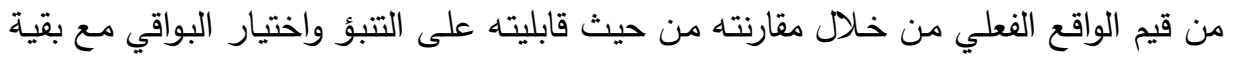
النماذج المقترحة.

3- تكون النماذج الخطية مستقره إذا كان كل من غير الخطية تكون الاستقرارية صعبة الحصول . 


\section{المصادز}

البد راني ،ظافر رمضان مطر (2002) "دراسـة في تثخيص نظم السيطرة التصادفية مع إثـارة خاصـة إلى أسلوب فضاء ألحالـه و الاستقرارية",أطروحة دكتوراه غير منشورة كلية علوم الحاسبات والرياضيات،جامعة الموصل.

الجبوري ،نهاد شريف خلف (2005)"دراسـة الاستقرارية في بعض النماذج غير الخطية مع تطبيق" رسالة ماجستير غير منشورة ،كلية التربية،جامعة تكريت.

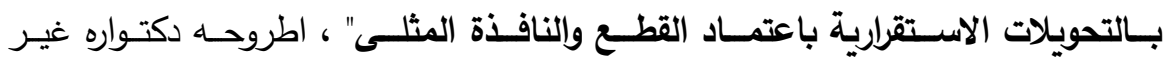
منشورة،كلية الاداره والاقتصاد ، جامعة بغداد.

$$
\text { الثر جبي ، قاسم عبده علي (2003) "المقدرات الحصينة لنماذج المختلطة(1,1) }
$$

ARMA والاقتصاد الجامعة المستتصرية . .

$$
\text { العجيلي ،سندس نوري شكر (2002) "بناء نموذج تصادفي لعدد الإصابات بمرض التدرن }
$$

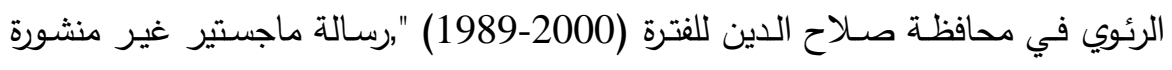$$
\text { كلية التربية بنات ،جامعة تكريت . }
$$

المهداوي ،هيفاء جعفر (1991) "الانحدار الذاتي غير المستقر ذو الدرجات الدنيا " أطروحة دكتوراه غير منشورة ،كلية الاداره والاقتصاد ،الجامعة المستتصرية.

[7] Bongeral, P. and Picard, N. (1992) "Stationaity of GARCH processes and some non negative time series " J. Econometrics 52,115-127.

[8] Brockwell, P.J. \& Stramer, O. and Tweedie, R.L. (1996) " Existences of stability of continuous time threshold ARMA processes " J. statistical since, Taipei, Vol. 6, pp. 715.

[9] Homiltion, James D.,(1994) "Time Series Analysis " , Published by princetion University Press, U.S.A.

[10] Husmeier, D. \& Taylor, J. G. (1997) " predicting Conditional Probability densities of stationary stochastic time series " Neural Networks, 10(3), pp.479-497. 
[11] Liu, J. \& susko, E. (1992) "On strict stationarity and ergodicity non linear ARMA Model" J.Appl. probab.29: pp.363-373.

[12] Manuca, R. \& Savit, R. (1996)"Stationarity and non statioarity

[13] Meyn, S. P. \& Tweedie, R.L. (1993) " Markov chains and stochastic stability " springer - varlag, London.

[14] Ozaki,T.(1982) "Non linear time series stochastic processes and Dynamical system" Handbook of statistics, Vol.5,Itd.

[15] Ozaki,T.(1985) "Non linear time series models and Dynamical system" E.J. Hannan, P.R. Krishnaiah, M,M.Rao, eds., Handbook of statistics,Vol.5,pp.25-83.

[16] Ozaki, T. and Oda, H.(1987)"Non-linear time series model identification by Akaikes information criterion ".proc. IFAC workshop on Information and systems, compiegn, France, October 1997.

[17] Pettersson, S. \& Lennartson, B. (1996) " LMI approach for stability and robusness of hybrid systems" , in proc. Amer. Control conf., Albuquerque NM, pp. 1714-1718.

[18] Priestly, M.B.(1988) " Non linear and stationary time series analysis " London: ACADEMIC. Press.

[19] Schreiber. T. (1997) " Detecting and analysis non stationirty in a time series using non linear cross predictions " physical Review Letters 78.

[20] Tong . H. (1983) " Threshol Models in Non linear time series Analysis " Lecture notes in statistic No. 21, Now York.

[21] Tong, H. (1990) " Non linear Time series; A Dynamical system Approach ".Oxford University Press ,London.

[22] Tsay ,RS(1986)."Non linearity Tests for Time series "Biometrika,73,461-466.

[23] Witt, A. \& Kurths, J. \& Pikosvky, A. (1998), " Testing statinoarity in time series", physical Review E 58:1800-1810. 